\title{
Fasting glucose, bone area and bone mineral density: a Mendelian randomisation study
}

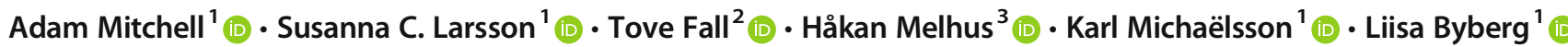

Received: 30 June 2020 / Accepted: 20 November 2020 / Published online: 2 March 2021

(C) The Author(s) 2021

\begin{abstract}
Aims/hypothesis Observational studies indicate that type 2 diabetes mellitus and fasting glucose levels are associated with a greater risk for hip fracture, smaller bone area and higher bone mineral density (BMD). However, these findings may be biased by residual confounding and reverse causation. Mendelian randomisation (MR) utilises genetic variants as instruments for exposures in an attempt to address these biases. Thus, we implemented MR to determine whether fasting glucose levels in individuals without diabetes are causally associated with bone area and BMD at the total hip.

Methods We selected 35 SNPs strongly associated with fasting glucose $\left(p<5 \times 10^{-8}\right)$ in a non-diabetic European-descent population from the Meta-Analyses of Glucose and Insulin-related traits Consortium (MAGIC) $(n=133,010)$. MR was used to assess the associations of genetically predicted fasting glucose concentrations with total hip bone area and BMD in 4966 men and women without diabetes from the Swedish Mammography Cohort, Prospective Investigation of Vasculature in Uppsala Seniors and Uppsala Longitudinal Study of Adult Men.

Results In a meta-analysis of the three cohorts, a genetically predicted $1 \mathrm{mmol} / \mathrm{l}$ increment of fasting glucose was associated with a $2 \%$ smaller total hip bone area $\left(-0.67 \mathrm{~cm}^{2}[95 \% \mathrm{CI}-1.30,-0.03 ; p=0.039]\right)$, yet was also associated, albeit without reaching statistical significance, with a $4 \%$ higher total hip BMD $\left(0.040 \mathrm{~g} / \mathrm{cm}^{2}[95 \% \mathrm{CI}-0.00,0.07 ; p=0.060]\right)$.

Conclusions/interpretation Fasting glucose may be a causal risk factor for smaller bone area at the hip, yet possibly for greater BMD. Further MR studies with larger sample sizes are required to corroborate these findings.
\end{abstract}

Keywords Bone area $\cdot$ Bone mineral density $\cdot$ Fasting glucose $\cdot$ Mendelian randomisation $\cdot$ Single nucleotide polymorphisms

\section{Abbreviations}

BMD Bone mineral density

DXA Dual energy x-ray absorptiometry

HRC Haplotype Reference Consortium

IVW Inverse-variance weighted

MAF Minor allele frequency

MAGIC Meta-Analyses of Glucose and Insulin-related traits Consortium

Adam Mitchell

Adam.mitchell@surgsci.uu.se

1 Department of Surgical Sciences, Orthopaedics, Uppsala University, Uppsala, Sweden

2 Department of Medical Sciences, Molecular Epidemiology, Uppsala University, Uppsala, Sweden

3 Department of Medical Sciences, Clinical Pharmacogenomics and Osteoporosis, Uppsala University, Uppsala, Sweden
MR Mendelian randomisation

PIVUS Prospective Investigation of the Vasculature of Uppsala Seniors

ROI Region of interest

SMC Swedish Mammography Cohort

SMCC Swedish Mammography Cohort - Clinical

ULSAM Uppsala Longitudinal Study of Adult Men

wGRS Weighted genetic risk score

\section{Introduction}

Observational studies have shown type 2 diabetes mellitus to be associated with a greater risk for hip fracture [1, 2], despite also being associated with a greater bone mineral density (BMD) [3]. Type 2 diabetes may therefore have other 


\section{Research in context}

\section{What is already known about this subject?}

- Type 2 diabetes mellitus is associated with a greater risk for hip fracture, greater bone mineral density (BMD) and smaller bone area

- Fasting glucose levels in individuals without type 2 diabetes are inversely associated with bone area in a dosedependent pattern

- It is unclear whether these associations are causal

\section{What is the key question?}

- Are fasting glucose levels causally associated with bone area and BMD at the total hip in individuals without diabetes?

\section{What are the new findings?}

- Genetic predisposition to higher fasting glucose may be a causal risk factor for smaller bone area at the hip

- This may also be the case for greater BMD, but the associations were weaker

\section{How might this impact on clinical practice in the foreseeable future?}

- These results highlight the potential causal relationship between fasting glucose levels and smaller bone area, which strengthens the understanding of the detrimental effects that characteristics of type 2 diabetes have on bone

detrimental effects resulting in a greater fracture risk. Potential mechanisms include weakened bone structure [4], diminished levels of bone turnover with greater bone loss [5], an increased risk of falling [6], or smaller bone area [7]. Bone size is integral for bone strength [8]. Specifically, bone strength is proportional to the fourth power of the radius, such that a doubling in cortex diameter will yield eightfold increments in mechanical resistance to bending and torsional loads [9]. Throughout life, bone expands as a compensation for agerelated loss in BMD [10]. We have previously shown, in a cross-sectional study using two Swedish cohorts (Swedish Mammography Cohort - Clinical [SMCC] and Uppsala Longitudinal Study of Adult Men [ULSAM]), that this increase in bone area may be less in individuals with type 2 diabetes [11]. We measured the total hip bone area, but others have also shown smaller bone area at the tibia [12] and radius [7] in those with type 2 diabetes. It is unclear which particular aspects of type 2 diabetes are responsible for the damaging effects on bone, as it is a complex disease characterised by both high blood glucose and complications with insulin action or secretion. In addition to type 2 diabetes, we also showed in our previous study that fasting glucose levels were inversely associated with bone area in a dose-dependent pattern [11].

To determine the causal effects on bone of type 2 diabetes or glucose levels in a non-diabetic population using traditional epidemiological approaches may be difficult due to a number of potential biases, including residual confounding and reverse causation [13]. To conduct a randomised trial where individuals would be randomised to certain levels of fasting glucose for many years to represent type 2 diabetes risk would be unethical and impossible. The Mendelian randomisation (MR) approach uses genetic data as instrumental variables to examine the effects of modifiable risk factors and various disease outcomes, particularly in observational data settings when confounding is a major concern [14]. Moreover, because allele assignment at meiosis is random and precedes the onset of disease, the risk of confounding is limited and there is no concern for the possibility of reverse causation [15]. Previous MR studies indicate that genetically determined fasting glucose levels [16] and type 2 diabetes risk [16, 17] increases BMD at the femoral neck, but no association was seen with risk of any fracture among adults $>18$ years [17].

No MR study to date has addressed the effect of fasting glucose on bone area, one potential mechanism underlying the association between type 2 diabetes and hip fracture. Several studies indicate that bone area at the hip is linked with the risk of hip fracture [18, 19], and it is therefore of clinical importance to investigate whether the observational association between higher fasting glucose concentrations and a smaller bone area at the hip - previously presented by our research group [11] - is causal. As the genetic instruments available for glucose concentrations were established among individuals without diabetes [20], and the genetically increased glucose concentrations will influence the outcome independent of type 2 diabetes status [21], we implemented an MR approach to determine whether fasting glucose levels are causally associated with total hip bone area, and to verify the association with total hip BMD, in individuals without 
diabetes in three Swedish cohorts. The observational associations were based on two of these three cohorts [11].

\section{Methods}

Study samples All study populations were restricted to individuals without diabetes in the three Swedish cohorts detailed below. We defined diabetes according to the ADA and WHO criteria: fasting plasma glucose concentrations $\geq 7.0 \mathrm{mmol} / \mathrm{l}$ and/or self-reported diabetes with or without treatment with oral hypoglycaemic agents or insulin.

SMCC The Swedish Mammography Cohort (SMC) was established during 1987-1990. Between November 2003 and October 2009, a randomly selected sub-cohort (SMCC) of 5022 women living in the city of Uppsala, Sweden, underwent dual energy $\mathrm{x}$-ray absorptiometry (DXA) measurements, provided morning fasting blood samples, had height and weight measurements taken, and completed a medical and lifestyle questionnaire [22]. Of these, 3945 women had complete information on fasting glucose, diabetes status, genetic data and DXA measurements and were thus included in our analyses. Genotyping in the SMCC was performed using the Illumina GSAMD-24v1-0 20011747 A1 BeadChip, USA and SNPs were imputed up to Haplotype Reference Consortium (HRC) v1.1 and 1000 Genomes project phase 3 . The results were then analysed using the software GenomeStudio 2.0.3 from Illumina, USA. The sample success rate was $\geq 98 \%$. The SMCC is managed by the Swedish Infrastructure for Medical Population-based Life-course and Environmental Research (www. simpler4health.se).

Prospective investigation of the vasculature of Uppsala seniors Between 2001 and 2004, all 70-year-old residents of Uppsala, Sweden, were invited to participate in a health survey and clinical assessment [23]. Of 2025 invited, 1016
(50.2\%) participated in the baseline assessment. From these, 691 participants had complete information on fasting blood glucose (converted to plasma concentrations) [24], diabetes status, genetic data and DXA measurements and were included in our analyses. Genotyping in the Prospective Investigation of the Vasculature of Uppsala Seniors (PIVUS) was performed using Illumina OmniExpress+Metabochip, USA, quality controlled and imputed up to the HRC panel using the software IMPUTE (https://mathgen.stats.ox.ac.uk/ impute/impute_v1_html). The sample success rate was $98.8 \%$ and the reproducibility $100 \%$ according to duplicate analysis of $2.4 \%$ of the genotypes.

ULSAM In 1970, all men born between 1920 and 1924, living in the county of Uppsala, Sweden, were invited to a take part in a health survey [25]. The men who participated were regularly re-examined, and the current analyses were based on the fifth examination cycle in 2003-2005, when 952 men were invited for examination and 526 of them were examined (mean age 82 years). Of these men, 360 had complete information on fasting glucose, diabetes status, genetic data and DXA measurements and were thus included in our analyses. Genotyping was performed using Illumina Omni2.5+ Metabochip and GenomeStudio 2010.3, USA and imputed up to the HRC panel using the software IMPUTE. The sample success rate was $\geq 99 \%$, minor allele frequency (MAF $<5 \%$ ) or $\geq 95 \%$ (MAF $\geq 5 \%$ ).

This study complies with the Declaration of Helsinki. The ethics committee of Uppsala University approved the studies (ethical approval numbers 2010/0148-32 [Stockholm] and 2019-02125 [Uppsala]). All participants provided their informed consent.

Bone area and BMD Bone area $\left(\mathrm{cm}^{2}\right)$ and BMD $\left(\mathrm{g} / \mathrm{cm}^{2}\right)$ of the total hip and femoral shaft were measured by DXA (DPX Prodigy, Lunar, Madison, WI, USA). All measurements in all three cohorts were performed on the same DXA machine by the same experienced and accredited DXA x-ray nurse.
Fig. 1 DXA image of dual femur and the total hip in an adult male. The total area within the blue lines defines the total hip area ROI. This is the standard output from the Lunar Prodigy DXA scanner [11]

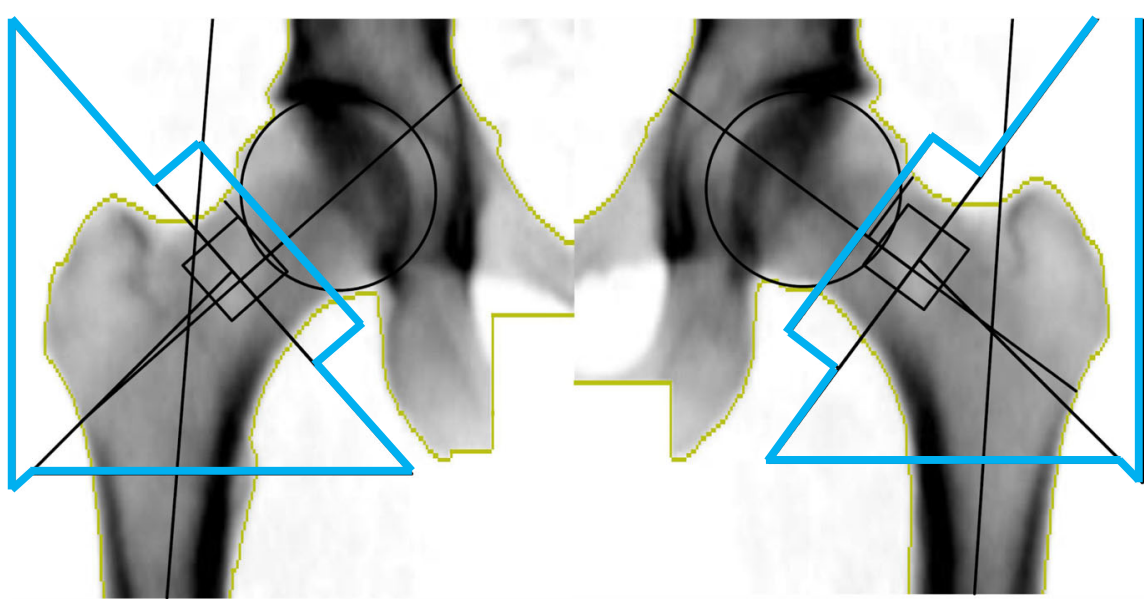




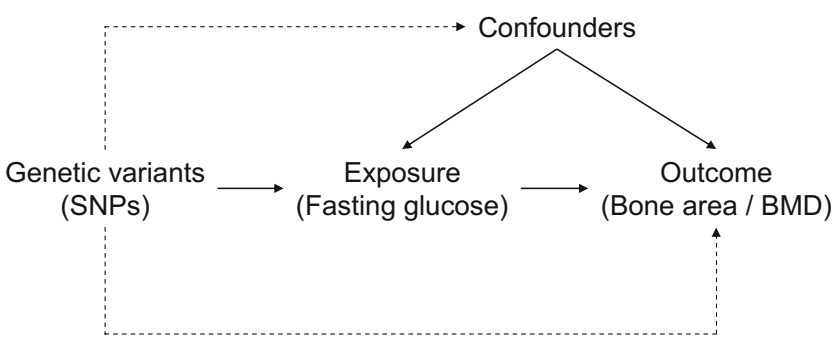

Fig. 2 The instrumental variables assumptions for MR. The three assumptions are: (1) the genetic variants are robustly associated with the exposure; (2) they are not associated with confounders of the exposure-outcome relationship; and (3) they have no association with the outcome except through their association with the exposure. The dashed lines represent pathways that violate the assumptions

The hip was set in a standard position by a fixed position of the knee, ankle and foot, to ensure that area did not vary due to rotational differences, and each scan was checked before it was accepted. The total hip area region of interest (ROI) was defined as the total area within the blue lines, corresponding to the femoral neck, Ward's area, trochanter, and femoral shaft ROIs (Fig. 1) [11]. The ROI was adjusted to the same location for each participant if needed ( $<0.5 \%$ of scans). The precision error from the DXA was $<1 \%$ for BMD and bone area at the total hip. To quantify differences in bone area and BMD, we calculated a percentage difference by dividing the $\beta$ estimate generated from the meta-analysed inverse-variance weighted (IVW) regression models by the mean value of either bone area or BMD multiplied by 100 .

SNP selection As instrumental variables, we selected the 36 SNPs associated with fasting glucose concentrations at a genome-wide significance threshold $\left(p<5 \times 10^{-8}\right)$ in a population without diabetes of European descent $(n=133,010)$ from the Meta-Analyses of Glucose and Insulin-related traits Consortium (MAGIC) [20]. One SNP (rs10747083) was associated with fasting glucose in the opposite direction compared with that in MAGIC in all three included Swedish cohorts and was excluded from subsequent analysis as it was not deemed a robust instrument, leaving 35 SNPs as instrumental variables in the present analyses. All 35 SNPs were available in the three cohorts and were independent (linkage disequilibrium $\mathrm{R}^{2}<0.01$ in European population).

MR analysis The MR approach was used to obtain quantitative estimates of the causal effects of fasting glucose on total hip bone area and BMD, based on the assumptions that the genetic variants used as instrumental variables: (1) are associated with the exposure (fasting glucose); (2) are not associated with any confounders of the exposure-outcome association; and (3) are associated with bone area and BMD through the exposure only and not through any alternative causal pathway ensuring a lack of pleiotropy (Fig. 2).
Linear regression models, adjusted for age and genetic principal components (SMCC $n=10$, PIVUS $n=2$, ULSAM $n=4)$, were applied to estimate the association between each SNP and bone area and BMD at the total hip. In the primary analysis, the SNP-glucose and SNP-bone outcome $\beta$ coefficients were used to compute estimates of the associations of fasting glucose with the bone outcomes using the IVW method [26], first using fixed effects and then with random effects [27]. The MR estimates ( $\beta$ coefficients and standard errors) for the associations between genetically predicted fasting glucose and the outcomes computed from each of the three cohorts were then combined in a metaanalysis using the metan package for Stata (https://raw. github.com/remlapmot/mrrobust/master/).

To explore the robustness of the MR results we conducted analysis using the weighted median, which can provide a consistent estimate of the causal effect even when up to $50 \%$ of the genetic variants are invalid instruments [28]. We applied MR-Egger regression [29] methods using the mrrobust package [30] to identify and control for bias due to directional pleiotropy. Pleiotropy was evaluated based on the intercept obtained from the MR-Egger analysis [31]. To identify any potential outliers and examine the extent of horizontal pleiotropy, we applied the MR-Pleiotropy RESidual Sum and Outlier (PRESSO) method [32] using the MR-PRESSO package in R (https://github.com/rondolab/MR-PRESSO).

In sensitivity analyses, we used multivariable MR analysis to adjust for genetically predicted height [33] and BMI because of the known effects of height and BMI [34] on bone size and diabetes risk, and also removed SNP (rs7651090, for human gene $I G F 2 B P 2$ ) due to the known effects of IGF binding proteins on bone health. We then performed the above main analyses also including those with type 2 diabetes in our cohorts (total $n=4234$ in SMCC, 783 in PIVUS, 443 in ULSAM) and using sex-specific $\beta$ estimates for the associations of the SNPs with fasting glucose (accessed, 20 August 2020 from https://www.magicinvestigators.org/downloads/) and total hip bone area and BMD in our cohorts. A weighted genetic risk score (wGRS) was generated using the 35 SNPs and the $\beta$ estimates from the MAGIC consortium genome-wide association study data, and we conducted a onesample MR using the wGRS as the instrumental variable to estimate its association with bone area and BMD using the Wald ratio method ( $95 \%$ CI calculated using the delta method) and additionally with further adjustment for BMI and height. Statistical analyses were performed in Stata MP 15 (StataCorp, College Station, TX, USA) and R, partly using resources provided by SNIC-SENS (a SNIC project with the purpose of providing secure handling of sensitive data [such as human genomic data] to the research community) through the Uppsala Multidisciplinary Center for Advanced Computational Science (UPPMAX). A $p$ value of $<0.05$ was considered statistically significant. 
Table 1 Characteristics of the study population in the three cohorts by sex

\begin{tabular}{lcccc}
\hline \multirow{2}{*}{ Characteristic } & SMCC $(n=3945)$ & \multicolumn{2}{l}{ PIVUS } & \multirow{2}{*}{ ULSAM $(n=360)$} \\
\cline { 2 - 3 } & & Women $(n=359)$ & Men $(n=332)$ & \\
\hline Age (years) & $67.18 \pm 6.55$ & $70.24 \pm 0.28$ & $70.10 \pm 0.13$ & $81.69 \pm 0.98$ \\
BMI $\left(\mathrm{kg} / \mathrm{m}^{2}\right)$ & $25.75 \pm 4.20$ & $26.67 \pm 4.40$ & $26.90 \pm 3.60$ & $25.71 \pm 3.25$ \\
Weight $(\mathrm{kg})$ & $68.87 \pm 11.73$ & $69.36 \pm 12.47$ & $82.40 \pm 12.21$ & $76.75 \pm 10.59$ \\
Height $(\mathrm{cm})$ & $163.54 \pm 6.05$ & $161.63 \pm 5.54$ & $175.64 \pm 6.35$ & $172.71 \pm 5.78$ \\
Total hip bone area $\left(\mathrm{cm}^{2}\right)$ & $32.50 \pm 2.17$ & $32.91 \pm 2.10$ & $38.75 \pm 2.35$ & $39.16 \pm 2.70$ \\
Total hip BMD $\left(\mathrm{g} / \mathrm{cm}^{2}\right)$ & $0.91 \pm 0.13$ & $0.87 \pm 0.13$ & $1.02 \pm 0.15$ & $0.96 \pm 0.16$ \\
Fasting glucose $(\mathrm{mmol} / \mathrm{l})$ & $5.15 \pm 0.53$ & $5.50 \pm 0.55$ & $5.60 \pm 0.60$ & $5.53 \pm 0.58$ \\
\hline
\end{tabular}

Data are presented as mean \pm SD

In PIVUS, fasting glucose samples were converted from whole blood to plasma concentrations

\section{Results}

The characteristics of participants in each of the three Swedish cohorts are presented in Table 1. The mean weight, height, bone area and BMD were higher in both PIVUS and ULSAM compared with SMCC, as men were present in these cohorts, whereas SMCC comprised only women. Fasting glucose levels were also higher in PIVUS and ULSAM which may be explained by the older age in these cohorts compared with SMCC.

In the SMCC, PIVUS and ULSAM cohorts, the 35 SNPs used in our analyses together explained 4\% (adjusted $\mathrm{r}^{2}=$ 0.045 ), 4\% (adjusted $r^{2}=0.035$ ) and 12\% (adjusted $r^{2}=$ 0.119 ), respectively, of the variance in fasting glucose concentrations in participants without diabetes. The association of each SNP with bone area and BMD in each cohort, along with the $\beta$ estimates for the associations of the SNPs with fasting glucose concentrations, can be found in electronic supplementary material (ESM) Figs 1-6 and ESM Tables 1-3. There was low sample overlap (0.79\%) with PIVUS and ULSAM. SMCC, the largest cohort, is not included in MAGIC.

In conventional MR analysis, a $1 \mathrm{mmol} / \mathrm{l}$ higher genetically predicted fasting glucose concentration was associated with
$2 \%$ smaller total hip bone area $\left(-0.67 \mathrm{~cm}^{2}[95 \% \mathrm{CI}-1.30\right.$, $-0.03 ; p=0.039]$ ) based on meta-analysis of estimates from the three cohorts using the fixed effects IVW method (Fig. 3). In contrast to bone area, a $1 \mathrm{mmol} / 1$ higher genetically predicted fasting glucose concentration was associated, albeit without reaching statistical significance, with $4 \%$ higher total hip $\operatorname{BMD}\left(0.040 \mathrm{~g} / \mathrm{cm}^{2}\right.$ [95\% CI $\left.\left.-0.00,0.07 ; p=0.060\right]\right)$ in metaanalysis of estimates from the three cohorts (Fig. 4), although the precision in the estimate was low. Results were consistent in sensitivity analyses using the random effects IVW and weighted median methods (ESM Figs 7, 8).

We tested for pleiotropy using MR-Egger and MRPRESSO. The MR-Egger analysis did not provide evidence of pleiotropy in the analysis of bone area or BMD (Table 2) and the causal estimate was in the same direction as the primary analysis (Figs 5, 6). The MR-PRESSO method did not detect any outliers, but the global test $p$ value was 0.0129 in the BMD analysis based on ULSAM data indicating there may be some horizontal pleiotropy in that specific analysis. We found no evidence of heterogeneity in the primary analysis using the IVW method (ESM Table 4). Additional sensitivity analysis adjusting for the genetic effect of height and removing SNP rs7651090 did not affect the results essentially
Fig. 3 Meta-analysis of glucose variants to bone area in SMCC, PIVUS and ULSAM

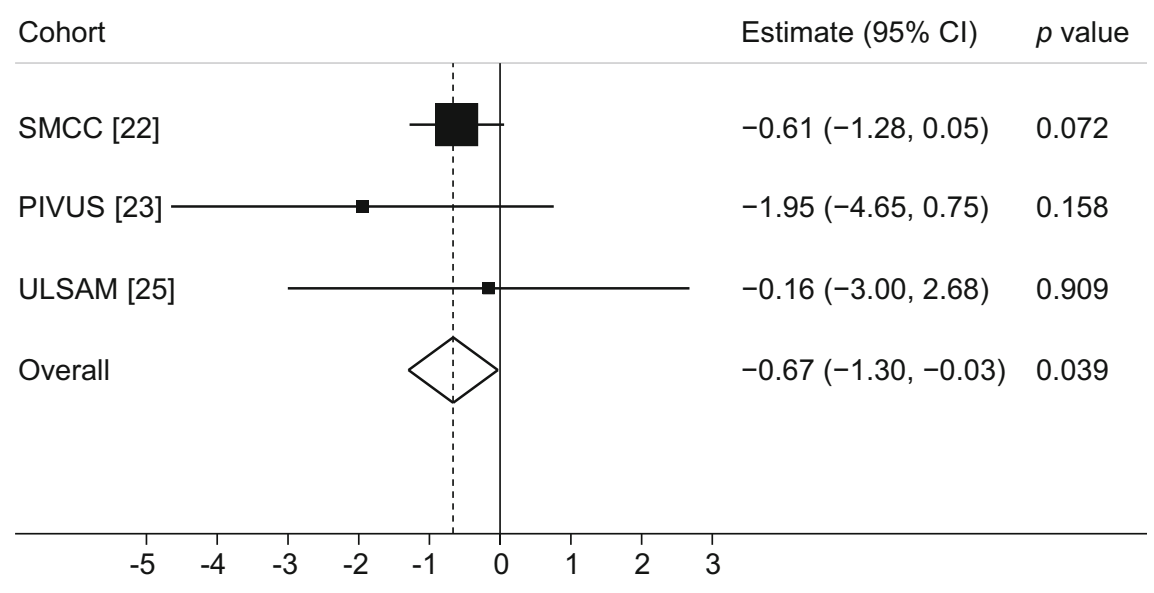


Fig. 4 Meta-analysis of glucose variants to BMD in SMCC, PIVUS and ULSAM

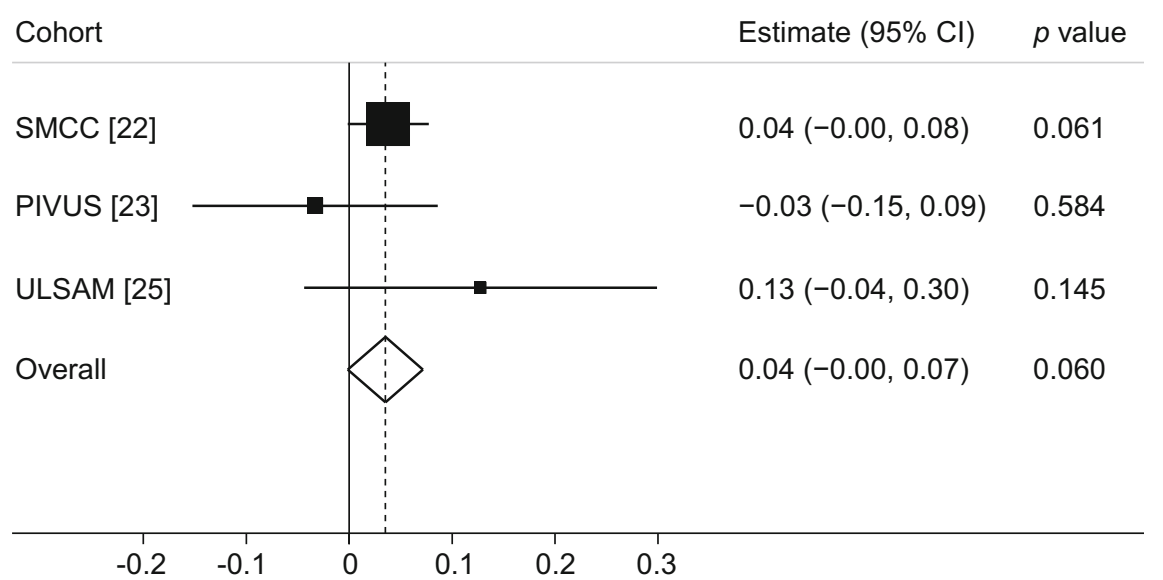

(ESM Figs 9-12) and neither did BMI (ESM Figs 13, 14). Including also those with type 2 diabetes in our cohorts did not change the interpretation of our results (ESM Figs 15, 16). Performing the analysis using sex-specific $\beta$ estimates gave similar results to the main analysis (ESM Figs 17, 18). In the one-sample MR analysis using the wGRS, estimates were once again in the same direction as the primary analysis (ESM Figs 19, 20) even with further adjustment for height and BMI (ESM Figs 21, 22). The resulting F-statistics were 196, 40 and 50 in SMCC, PIVUS and ULSAM, respectively (ESM Table 5).

\section{Discussion}

To the best of our knowledge, this is the first MR study using multiple genetic variants for fasting glucose and analysing their effect on bone area and BMD. Among men and women without diabetes in three Swedish cohorts, we found evidence that genetically increased fasting glucose levels were associated with smaller total hip bone area. The previously observed association with a greater BMD [16] was also observed in our study. We observed no evidence for directional pleiotropy. These results are partly based on the same population from SMCC and ULSAM where we previously observed that type

Table 2 MR-Egger tests for the presence of pleiotropy affecting the assessment of the effects of fasting glucose on the outcomes bone area and BMD of the total hip

\begin{tabular}{llcll}
\hline Outcome & Cohort & $\beta$ & SE & $p$ value \\
\hline Total hip bone area & SMCC & 0.197227 & 0.179006 & 0.271 \\
Total hip bone area & PIVUS & -0.003845 & 0.0691082 & 0.956 \\
Total hip bone area & ULSAM & -0.055295 & 0.0718004 & 0.441 \\
Total hip BMD & SMCC & 0.0006225 & 0.0010413 & 0.550 \\
Total hip BMD & PIVUS & 0.0023567 & 0.0031256 & 0.451 \\
Total hip BMD & ULSAM & 0.0041666 & 0.0056075 & 0.457 \\
\hline
\end{tabular}

2 diabetes and higher fasting glucose levels were associated with smaller bone area and greater BMD at the total hip [11].

Major strengths of the MR approach are that reverse causation bias is avoided because genetic variants are fixed at conception, and confounding is reduced by the use of genetic variants as proxies for the exposure representing the lifelong exposure of fasting glucose concentrations [35]. Our study population consisted of both men and women, and all DXA measurements in all three cohorts were taken by the same $\mathrm{X}$ ray nurse using the same DXA scanner, thereby increasing the reliability and reducing variance in our outcome measure. Magnification error from DXA scans can have direct effects on estimated bone area [36]. The Lunar Prodigy DXA scanner used in this study reduces the substantial magnification error by using a narrow fan-beam along the axis of measurement compared with other scanners which use a wide-angle fanbeam $[36,37]$. This reduces the impact of an individual's body weight which may otherwise affect the bone area measurement. The narrow fan-beam, high resolution, automatic location of the bone, and centring of the scan around the bone providing precise automatic edge detection combine to give an improved measurement that does not require any scout scans and is less dependent on the exact positioning of the femur in the beam [36, 37]. In all cohorts, we were able to clinically categorise individuals as having diabetes based on fasting glucose samples in combination with self-reported diagnosis and medication use, so that they could be excluded from the main analysis. The 35 genetic variants increase the fasting glucose levels, explaining $4-12 \%$ of the variance in our studies, and will therefore influence the outcome even if the type 2 diabetes status remains fixed for all participants in the analysis [21]. For a genetic instrument to be valid for a dichotomous exposure, such as type 2 diabetes, there needs to be a strict stepwise threshold at the cut-off point, and the interpretation of such an exposure is less straightforward than that of a continuous exposure [21]. Furthermore, the genetic instruments for type 2 diabetes may be influenced by treatment, and genetic instruments for insulin explain a very low 
Fig. 5 Meta-analysis of glucose variants to bone area in SMCC, PIVUS and ULSAM from MREgger test

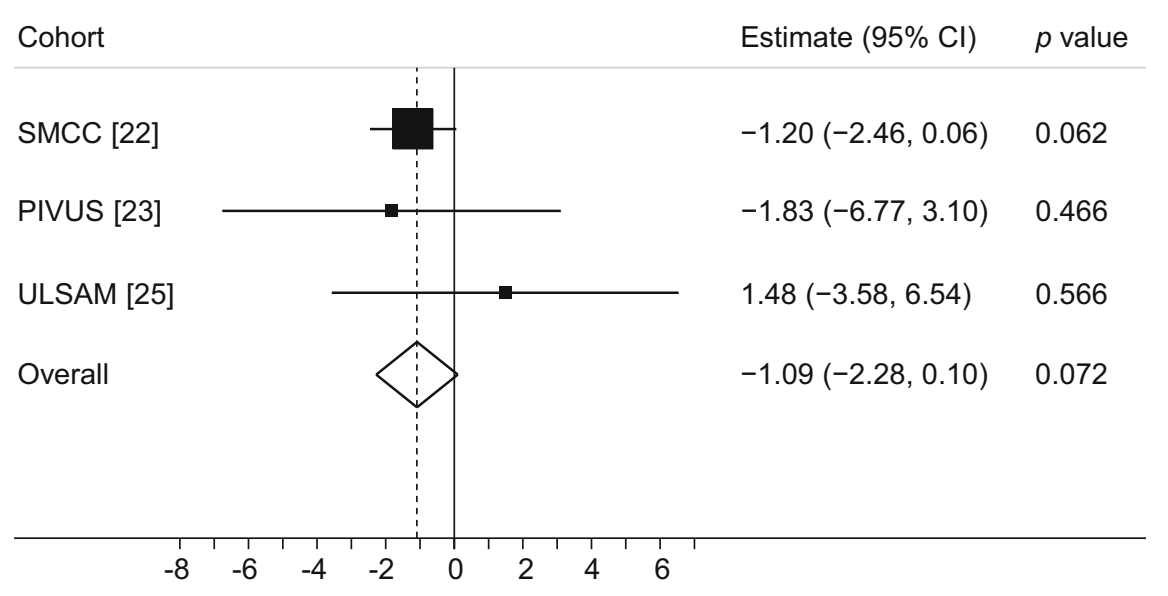

proportion of the variance in insulin concentrations [20]. Although heritability for type 2 diabetes is high, using genetic variants for fasting glucose concentrations as an instrument may therefore provide more specific insight into the mechanisms of glucose on bone area, also when explored in a population without diabetes [21]. Pleiotropic effects of the type 2 diabetes instrument is a concern since it includes obesityrelated SNPs, which increase the risk of diabetes through increasing BMI. For fracture-related outcomes, this could pose an additional problem by effects acting in opposing directions, as suggested by prior results showing type 2 diabetes to be associated with higher fracture risk and a high body weight with a lower fracture risk. Nevertheless, conducting the analysis among all participants showed results of the same direction and magnitude as the main analysis, indicating no large influence of potential collider bias after restriction to those with type 2 diabetes. The main limitation of our study is the limited study size, since MR analyses generally require large numbers of individuals. However, we were able to observe statistically significant results for the association between fasting glucose and bone area, and results were consistent using both two-sample MR and one-sample MR designs. We hope future studies and consortia will explore and present results also for bone area. A previous two- sample MR study [16], using summary level data of BMDassociated genetic variants from European and East Asian ancestry [38] among up to 83,894 individuals, reported that genetically increased fasting glucose increased femoral neck BMD and, albeit without reaching statistical significance, lumbar spine BMD. The authors suggested that diabetes may have different effects on cortical and trabecular bone. The total hip, used in our study, consisted of both cortical and trabecular bone and we found similar effects on BMD in that area, although lacking statistical power due to a small sample size. Another MR study found no association of genetically increased fasting glucose and type 2 diabetes on the risk of any fracture among adults $>18$ years [17]. Fragility fractures such as hip fractures occurring among older individuals may have a different aetiology than other types of fractures occurring among younger individuals, and to separate by fracture type may be crucial. Unfortunately, valid hip fracture information is, to date, lacking from large consortia. Inferring causality from MR analyses relies on several assumptions, one being the assumption that the genetic variants used as instruments are strongly associated with the risk factor. In a two-sample MR setting we used several genetic variants strongly associated with fasting glucose $\left(p<5 \times 10^{-8}\right)$ in a previous meta-analysis of genome-wide association studies of non-
Fig. 6 Meta-analysis of glucose variants to BMD in SMCC, PIVUS and ULSAM from MREgger test

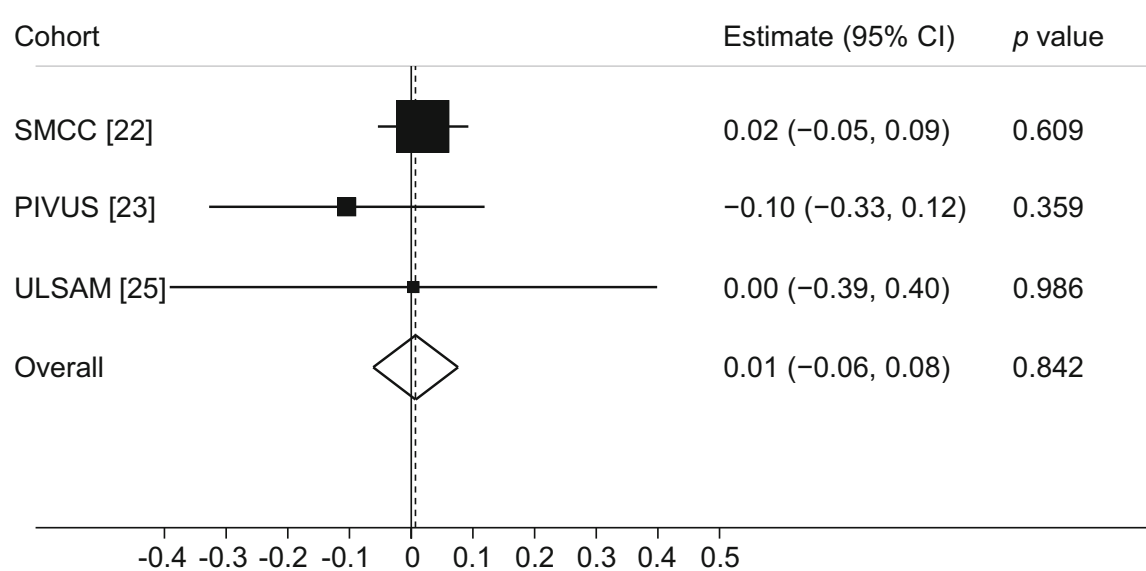


diabetic individuals [20], which would ensure that any bias from weak instruments is towards the null [39] and therefore increase the statistical power [26]. The instrument explained 4 $12 \%$ of the variation in fasting glucose concentrations in our populations, which is high compared with many traits. Further, results were in the same direction as in our main analysis when we applied the weighted median method, where the estimate remains consistent when up to $50 \%$ of the SNPs are invalid instruments [27], suggesting robust estimates. The overlap between our outcome cohorts and the MAGIC consortium data was very low and the F-statistics for our individual cohorts were high when assessing the association between the wGRS and fasting glucose levels, indicating that any bias would be towards the null.

MR analyses further rely on the assumption of absence of pleiotropy, which can occur when a genetic instrument (SNP) affects multiple phenotypes [40]. Dependent on the type of pleiotropy present, it can lead to biased estimates. Horizontal pleiotropy occurs when a genetic variant affects more than one phenotype on separate pathways [41], whereas vertical pleiotropy, also known as mediated pleiotropy, occurs when a genetic variant affects other phenotypes downstream from the exposure, on the causal pathway to the outcome [42]. In our case, type 2 diabetes might be one such mediating phenotype, being downstream of fasting glucose and also associated with bone phenotypes. Horizontal pleiotropy can lead to bias in an MR study [43] whereas vertical pleiotropy is of less concern. We cannot entirely exclude the possibility that the SNPs used as instruments in the present study may affect bone area and BMD through mechanisms other than their effects on fasting glucose. However, we did not find any evidence of horizontal or vertical pleiotropy using the MR-Egger approach. Our results also remained consistent when we adjusted for the genetic effects of height [33] and BMI [34] in a sensitivity analysis. Height has been shown to directly affect both bone size and the risk of diabetes [44].

We did not find any evidence of SNPs being outliers or effect change caused by any SNP outliers, evaluated using MR-PRESSO [32], although there may have been some pleiotropy in the ULSAM BMD analysis. Due to the known effects of IGF binding proteins on bone health [45], we removed SNP (rs7651090, for human gene IGF2BP2) in an additional sensitivity analysis but this did not affect our estimates. Another potential source of bias in MR analyses is population stratification and population heterogeneity [46], but this was reduced in our study because our genetic instruments and outcomes came from European populations and all our outcome populations were based in Sweden. We also adjusted for genetic principal components. However, this may limit the generalisability of our results to other ethnicities.

Bone size increases with advancing age via periosteal apposition to compensate for the losses in BMD in order to preserve bone strength [47]. The bending strength of bone and resistance to fracture is strongly related to bone diameter and small differences in area will result in large differences in strength [48]. It has been reported that increases in bone cortex diameter increases resistance to bending, torsional and compressive loads [49]. This suggests that the smaller bone area, seen with higher glucose concentrations, will yield a comparably lower bending resistance and strength, resulting in a bone that is more susceptible to fracture. The risk of incident hip fracture in older white women from the USA has been shown to be higher with lower composite indices of femoral neck strength [8].

The relationship between bone and energy metabolism is complex and may be bidirectional. A previous study found that each one SD increase in genetically estimated heel BMD (equivalent to $0.14 \mathrm{~g} / \mathrm{cm}^{2}$ ) was associated with an $8 \%$ higher risk of type 2 diabetes, assessed using an MR approach [50]. Consequently, it could be of interest to conduct a bidirectional MR study, although a major limitation is the low number of genetic variants reported for bone area. A study with large power could assess this direction of association.

By combining data from three separate cohorts in a twosample MR design, our study provided evidence that fasting glucose may be a causal risk factor for smaller bone area at the hip. We found suggestive evidence that fasting glucose may also lead to increased BMD. If other larger cohorts or consortia with DXA measurements of the hip, such as the UK Biobank, presented their bone area measurements, future MR studies could utilise the larger sample sizes required to corroborate these findings.

Supplementary Information The online version contains peer-reviewed but unedited supplementary material available at https://doi.org/10.1007/ s00125-021-05410-w.

Acknowledgements The computations were performed on the Bianca cluster provided by SNIC-SENS through the Uppsala Multidisciplinary Center for Advanced Computational Science (UPPMAX).

Data availability The data codes used to generate the results in this study are available from the corresponding author upon reasonable request. Data are not freely available, but the cohorts can be contacted to request access. SMCC: https://www.simpler4health.se/; PIVUS: https://www. medsci.uu.se/pivus; ULSAM: https://www.pubcare.uu.se/ulsam/

Funding Open access funding provided by Uppsala University. This work was supported by grants from the Swedish Research Council, grant numbers 2015-05997 and 2015-03527. We acknowledge SIMPLER (www.simpler4health.se) for the provision of facilities and experimental support. SIMPLER receives funding through the Swedish Research Council under grant number 2017-00644.

Authors' relationships and activities The authors declare that there are no relationships or activities that might bias, or be perceived to bias, their work.

Contribution statement The study was conceived and designed by AM, SCL, TF, KM and LB. AM and SCL analysed the data. All authors contributed to the interpretation of the data and critical revision of the 
manuscript. AM, SCL and LB drafted the manuscript. All authors read and approved the final manuscript. AM takes responsibility for the integrity of the data analysis.

Open Access This article is licensed under a Creative Commons Attribution 4.0 International License, which permits use, sharing, adaptation, distribution and reproduction in any medium or format, as long as you give appropriate credit to the original author(s) and the source, provide a link to the Creative Commons licence, and indicate if changes were made. The images or other third party material in this article are included in the article's Creative Commons licence, unless indicated otherwise in a credit line to the material. If material is not included in the article's Creative Commons licence and your intended use is not permitted by statutory regulation or exceeds the permitted use, you will need to obtain permission directly from the copyright holder. To view a copy of this licence, visit http://creativecommons.org/licenses/by/4.0/.

\section{References}

1. Janghorbani M, Feskanich D, Willett WC, Hu F (2006) Prospective study of diabetes and risk of hip fracture: the Nurses' Health Study. Diabetes Care 29(7):1573-1578. https://doi.org/10.2337/dc060440

2. Fan Y, Wei F, Lang Y, Liu Y (2016) Diabetes mellitus and risk of hip fractures: a meta-analysis. Osteoporos Int 27(1):219-228. https://doi.org/10.1007/s00198-015-3279-7

3. Ma L, Oei L, Jiang L et al (2012) Association between bone mineral density and type 2 diabetes mellitus: a meta-analysis of observational studies. Eur J Epidemiol 27(5):319-332. https://doi.org/10. 1007/s10654-012-9674-x

4. Vestergaard P (2007) Discrepancies in bone mineral density and fracture risk in patients with type 1 and type 2 diabetes-a metaanalysis. Osteoporos Int 18(4):427-444. https://doi.org/10.1007/ s00198-006-0253-4

5. Hygum K, Starup-Linde J, Harslof T, Vestergaard P, Langdahl BL (2017) MECHANISMS IN ENDOCRINOLOGY: Diabetes mellitus, a state of low bone turnover - a systematic review and meta-analysis. Eur J Endocrinol 176(3):R137-r157. https://doi. org/10.1530/eje-16-0652

6. Schwartz AV, Hillier TA, Sellmeyer DE et al (2002) Older women with diabetes have a higher risk of falls: a prospective study. Diabetes Care 25(10):1749-1754. https://doi.org/10.2337/diacare. 25.10.1749

7. Samelson EJ, Demissie S, Cupples LA et al (2018) Diabetes and deficits in cortical bone density, microarchitecture, and bone size: Framingham HR-pQCT study. J Bone Miner Res Off J Am Soc Bone Miner Res 33(1):54-62. https://doi.org/10.1002/jbmr.3240

8. Karlamangla AS, Barrett-Connor E, Young J, Greendale GA (2004) Hip fracture risk assessment using composite indices of femoral neck strength: the Rancho Bernardo study. Osteoporos Int 15(1):62-70. https://doi.org/10.1007/s00198-003-1513-1

9. Hart NH, Nimphius S, Rantalainen T, Ireland A, Siafarikas A, Newton RU (2017) Mechanical basis of bone strength: influence of bone material, bone structure and muscle action. J Musculoskelet Neuronal Interact 17(3):114-139

10. Seeman E (2003) Periosteal bone formation-a neglected determinant of bone strength. N Engl J Med 349(4):320-323. https://doi. org/10.1056/NEJMp038101

11. Mitchell A, Fall T, Melhus H, Wolk A, Michaelsson K, Byberg L (2018) Type 2 diabetes in relation to hip bone density, area, and bone turnover in Swedish men and women: a cross-sectional study.
Calcif Tissue Int 103(5):501-511. https://doi.org/10.1007/s00223018-0446-9

12. Colleluori G, Aguirre L, Dorin R et al (2017) Hypogonadal men with type 2 diabetes mellitus have smaller bone size and lower bone turnover. Bone 99:14-19. https://doi.org/10.1016/j.bone.2017.03. 039

13. Nørgaard M, Ehrenstein V, Vandenbroucke JP (2017) Confounding in observational studies based on large health care databases: problems and potential solutions - a primer for the clinician. Clin Epidemiol 9:185-193. https://doi.org/10.2147/CLEP. S129879

14. Evans DM, Davey Smith G (2015) Mendelian randomization: new applications in the coming age of hypothesis-free causality. Annu Rev Genomics Hum Genet 16:327-350. https://doi.org/10.1146/ annurev-genom-090314-050016

15. Smith GD, Ebrahim S (2003) 'Mendelian randomization': can genetic epidemiology contribute to understanding environmental determinants of disease? Int J Epidemiol 32(1):1-22. https://doi. org/10.1093/ije/dyg070

16. Ahmad OS, Leong A, Miller JA et al (2017) A Mendelian randomization study of the effect of type-2 diabetes and glycemic traits on bone mineral density. J Bone Miner Res Off J Am Soc Bone Miner Res 32(5):1072-1081. https://doi.org/10.1002/jbmr.3063

17. Trajanoska K, Morris JA, Oei L et al (2018) Assessment of the genetic and clinical determinants of fracture risk: genome wide association and mendelian randomisation study. BMJ 362:k3225. https://doi.org/10.1136/bmj.k3225

18. Ahlborg HG, Nguyen ND, Nguyen TV, Center JR, Eisman JA (2005) Contribution of hip strength indices to hip fracture risk in elderly men and women. J Bone Miner Res Off J Am Soc Bone Miner Res 20(10):1820-1827. https://doi.org/10.1359/jbmr. 050519

19. Seeman E, Duan Y, Fong C, Edmonds J (2001) Fracture sitespecific deficits in bone size and volumetric density in men with spine or hip fractures. J Bone Miner Res Off J Am Soc Bone Miner Res 16(1):120-127. https://doi.org/10.1359/jbmr.2001.16.1.120

20. Scott RA, Lagou V, Welch RP et al (2012) Large-scale association analyses identify new loci influencing glycemic traits and provide insight into the underlying biological pathways. Nat Genet 44(9): 991-1005. https://doi.org/10.1038/ng.2385

21. Burgess S, Labrecque JA (2018) Mendelian randomization with a binary exposure variable: interpretation and presentation of causal estimates. Eur J Epidemiol 33(10):947-952. https://doi.org/10. 1007/s10654-018-0424-6

22. Michaelsson K, Wolk A, Byberg L, Mitchell A, Mallmin H, Melhus H (2017) The seasonal importance of serum 25hydroxyvitamin $\mathrm{D}$ for bone mineral density in older women. J Intern Med 281(2):167-178. https://doi.org/10.1111/joim.12563

23. Lind L, Fors N, Hall J, Marttala K, Stenborg A (2005) A comparison of three different methods to evaluate endothelium-dependent vasodilation in the elderly: the Prospective Investigation of the Vasculature in Uppsala Seniors (PIVUS) study. Arterioscler Thromb Vasc Biol 25(11):2368-2375. https://doi.org/10.1161/01. ATV.0000184769.22061.da

24. Kumar J, Ingelsson E, Lind L, Fall T (2015) No evidence of a causal relationship between plasma homocysteine and type 2 diabetes: a Mendelian randomization study. Front Cardiovasc Med 2:11. https://doi.org/10.3389/fcvm.2015.00011

25. Michaëlsson K, Lithell H, Vessby B, Melhus H (2003) Serum retinol levels and the risk of fracture. N Engl J Med 348(4):287-294. https://doi.org/10.1056/NEJMoa021171

26. Burgess S, Butterworth A, Thompson SG (2013) Mendelian randomization analysis with multiple genetic variants using summarized data. Genet Epidemiol 37(7):658-665. https://doi. org/10.1002/gepi.21758 
27. Burgess S, Bowden J, Fall T, Ingelsson E, Thompson SG (2017) Sensitivity analyses for robust causal inference from Mendelian randomization analyses with multiple genetic variants. Epidemiology (Cambridge, Mass) 28(1):30-42. https://doi.org/10. 1097/ede.0000000000000559

28. Bowden J, Davey Smith G, Haycock PC, Burgess S (2016) Consistent estimation in Mendelian randomization with some invalid instruments using a weighted median estimator. Genet Epidemiol 40(4):304-314. https://doi.org/10.1002/gepi.21965

29. Bowden J, Davey Smith G, Burgess S (2015) Mendelian randomization with invalid instruments: effect estimation and bias detection through Egger regression. Int J Epidemiol 44(2):512-525. https://doi.org/10.1093/ije/dyv080

30. Spiller W, Davies NM, Palmer TM (2018) Software application profile: mrrobust - a tool for performing two-sample summary Mendelian randomization analyses. Int J Epidemiol 48(3):684690. https://doi.org/10.1093/ije/dyy195

31. Burgess S, Thompson SG (2017) Interpreting findings from Mendelian randomization using the MR-Egger method. Eur J Epidemiol 32(5):377-389. https://doi.org/10.1007/s10654-0170255-x

32. Verbanck M, Chen CY, Neale B, Do R (2018) Detection of widespread horizontal pleiotropy in causal relationships inferred from Mendelian randomization between complex traits and diseases. Nat Genet 50(5):693-698. https://doi.org/10.1038/s41588-018-0099-7

33. Wood AR, Esko T, Yang J et al (2014) Defining the role of common variation in the genomic and biological architecture of adult human height. Nat Genet 46(11):1173-1186. https://doi.org/ 10.1038/ng.3097

34. Pulit SL, Stoneman C, Morris AP et al (2019) Meta-analysis of genome-wide association studies for body fat distribution in 694 649 individuals of European ancestry. Hum Mol Genet 28(1):166174. https://doi.org/10.1093/hmg/ddy327

35. Smith GD, Ebrahim S (2004) Mendelian randomization: prospects, potentials, and limitations. Int J Epidemiol 33(1):30-42. https://doi. org/10.1093/ije/dyh132

36. Mazess RB, Hanson JA, Payne R, Nord R, Wilson M (2000) Axial and total-body bone densitometry using a narrow-angle fan-beam. Osteoporos Int 11(2):158-166. https://doi.org/10.1007/p100004178

37. Bazzocchi A, Ponti F, Albisinni U, Battista G, Guglielmi G (2016) DXA: technical aspects and application. Eur J Radiol 85(8):14811492. https://doi.org/10.1016/j.ejrad.2016.04.004

38. Estrada K, Styrkarsdottir U, Evangelou E et al (2012) Genomewide meta-analysis identifies 56 bone mineral density loci and reveals 14 loci associated with risk of fracture. Nat Genet 44(5): 491-501. https://doi.org/10.1038/ng.2249
39. Burgess S, Davies NM, Thompson SG (2016) Bias due to participant overlap in two-sample Mendelian randomization. Genet Epidemiol 40(7):597-608. https://doi.org/10.1002/gepi.21998

40. Stearns FW (2010) One hundred years of pleiotropy: a retrospective. Genetics 186(3):767. https://doi.org/10.1534/genetics.110. 122549

41. Davey Smith G, Hemani G (2014) Mendelian randomization: genetic anchors for causal inference in epidemiological studies. Hum Mol Genet 23(R1):R89-R98. https://doi.org/10.1093/hmg/ ddu328

42. Hemani G, Bowden J, Davey Smith G (2018) Evaluating the potential role of pleiotropy in Mendelian randomization studies. Hum Mol Genet 27(R2):R195-R208. https://doi.org/10.1093/hmg/ ddy 163

43. Solovieff N, Cotsapas C, Lee PH, Purcell SM, Smoller JW (2013) Pleiotropy in complex traits: challenges and strategies. Nat Rev Genet 14(7):483-495. https://doi.org/10.1038/nrg3461

44. Wittenbecher C, Kuxhaus O, Boeing H, Stefan N, Schulze MB (2019) Associations of short stature and components of height with incidence of type 2 diabetes: mediating effects of cardiometabolic risk factors. Diabetologia 62(12):2211-2221. https://doi.org/10. 1007/s00125-019-04978-8

45. Liu H, Li D, Liu S, Liu Z, Li M (2018) Histochemical evidence of IGF2 mRNA-binding protein 2-mediated regulation of osteoclast function and adhesive ability. Histochem Cell Biol 149(4):343351. https://doi.org/10.1007/s00418-017-1629-6

46. Cardon LR, Palmer LJ (2003) Population stratification and spurious allelic association. Lancet 361(9357):598-604. https://doi.org/10. 1016/s0140-6736(03)12520-2

47. Ahlborg HG, Johnell O, Turner CH, Rannevik G, Karlsson MK (2003) Bone loss and bone size after menopause. N Engl J Med 349(4):327-334. https://doi.org/10.1056/NEJMoa022464

48. Beck TJ, Oreskovic TL, Stone KL et al (2001) Structural adaptation to changing skeletal load in the progression toward hip fragility: the study of osteoporotic fractures. J Bone Miner Res 16(6):1108 1119. https://doi.org/10.1359/jbmr.2001.16.6.1108

49. Davison KS, Siminoski K, Adachi JD et al (2006) Bone strength: the whole is greater than the sum of its parts. Semin Arthritis Rheum 36(1):22-31. https://doi.org/10.1016/j.semarthrit.2006.04. 002

50. Gan W, Clarke RJ, Mahajan A et al (2017) Bone mineral density and risk of type 2 diabetes and coronary heart disease: a Mendelian randomization study. Wellcome Open Res 2:68-68. https://doi.org/ 10.12688/wellcomeopenres.12288.1

Publisher's note Springer Nature remains neutral with regard to jurisdictional claims in published maps and institutional affiliations. 